\title{
Die Regierung der Anderen
}

\section{Von der mediterranen Todeslandschaft des europäischen Grenzregimes}

\section{The Government of the Others \\ On the Mediterranean Deathscape of Europe's Border Regime}

\section{Mareike Gebhardt, Münster}

Zusammenfassung: Der Beitrag nimmt eine politiktheoretisch-dispositivanalytische Perspektive ein. Er zeigt auf, inwiefern im mediterranen Grenzraum Europas nicht nur Mechanismen wirksam werden, die Migrant*innen verandern, sondern sie töten. Die letale VerAnderung vollzieht sich im europäischen Grenzregime innerhalb eines militärisch-humanitären Komplexes, in dem über Vergeschlechtlichung und Rassifizierung Migrant*innen mit spezifischen Affekten verkoppelt und dadurch de-/ humanisiert werden. Durch diese Regierung der Anderen sollen regressive Migrationspolitik und ein repressives Grenzregime plausibilisiert werden. Der Heterogenität des Grenzregimes Rechnung tragend diskutiert der Beitrag die diskursive Produktion des Anderen, dessen affektiv-performative Stabilisierung sowie die materiell-technologische Kultur der Grenze. Damit lehnt sich der Beitrag an die Arbeiten Foucaults zu Diskurs, Wahrheitsproduktion und Gouvernementalität an, fokussiert jedoch auf Theorien, die Foucaults Konzepte affekt- und geschlechtertheoretisch bzw. postkolonial reiterieren: Sara Ahmeds Konzept der affektiven Ökonomie, Judith Butlers Theorie der Betrauerbarkeit und Achille Mbembes Begriff der Vergrenzung. Dadurch werden die trauer- und nekropolitischen Aspekte des europäischen Grenzregimes nicht nur über politiktheoretische Perspektivierungen reflektiert, sondern durch einen rassismus- und geschlechterkritischen Blick ernst genommen.

Schlagwörter: Migration, affektive Ökonomie, Betrauerbarkeit, Ethnosexismus, Versicherheitlichung, Gouvernementalität 
Abstract: From the perspectives of political theory and dispositive analysis, the paper discusses how processes of othering work through the Mediterranean border space to eventually murder the migrant other. The lethal othering done by the European militaristic-humanitarian complex is part of an affective de-/humanization conducted via gendered and racialized processes. The government of the others leads to, strengthens, and justifies regressive migration policies and a repressive border regime. To acknowledge the heterogeneity of Europe's border regime, the paper focuses on three dimensions: the discursive production of the other, its affective-performative stabilization, and the material technologies that govern the border. In reference to Michel Foucault's works on discourse, knowledge production, and governmentality, the paper focuses on recent Foucauldian reiterations: Sara Ahmed's affective economies, Judith Butler's theory of grievability, and Achille Mbembes's concept of Vergrenzung ('borderization'). Hence, the paper not only scrutinizes the European border regime's necropolitics. Moreover, it argues for a thorough integration of feminist thought and postcolonial studies to the political theory of the border.

Keywords: Migration, affective economies, grievability, ethno-sexism, securitization, governmentality

„Indeed, for several years now, the European Union (EU) has actively converted the Mediterranean into a mass grave“. Nicholas de Genova, The Borders of "Europe" $(2017,3)$

\section{Einleitung: Die trauerpolitische Regierung der Anderen}

Es ist ein sozialwissenschaftlicher Gemeinplatz, dass die Konstruktion des Anderen das eigene Selbst herstellt und stabilisiert: $A b$-Grenzungen, ob nun materielle, rechtliche oder soziomentale, dienen der Produktion des Selbst. Diese Grenzziehung wird plausibiliert, indem der Andere auch als ein Fremder dargestellt wird. Julia Reuter $(2002,20)$ fasst dies im Begriff der „VerAnderung“ zusammen, der beschreibt „daß [sic] der Fremde als Anderer eben nicht einfach gegeben ist, auch niemals gefunden oder entdeckt, beschrieben oder beobachtet werden kann, sondern daß [sic] er durch seine Entdecker, Autoren und Beobachter mithervorgebracht wird“. Erst das Fremde im Anderen erweckt das Unheimliche in ihm: Er wird zur Gefahr des Eigenen und somit zu einem Sicherheitsrisiko, das zum Schutz des Eigenen eingedämmt werden muss.

Diese Logik wirkt auch im europäischen Grenzregime, das durch Kontroll-, Disziplinierungs- und Sicherheitstechnologien die Anderen im Grenz- 
raum regiert. ${ }^{1}$ In diesen (Regierungs-)Technologien der Grenze wirken Mechanismen der Sichtbarmachung, die die Anderen dem allzu hellen Licht der Überwachungsinstanzen aussetzen. Während seit dem Schengener Abkommen, das für Bürger*innen der Europäischen Union (EU) alle stationären Grenzkontrollen an den Binnengrenzen abschaffte und damit wesentlich dazu beitragen sollte, die Vorstellung Europas als „Raum der Freiheit, der Sicherheit und des Rechts“ (EUV Art. 3, Abs. 2) durchzusetzen, die Grenzen ,Europas` für deren Staatsbürger*innen weitestgehend unsichtbar sind, entfalten sie für die fremden Anderen eine grelle Sichtbarkeit im Namen der Versicherheitlichung: juridico-politische Repressionen, soziokulturelle Überfremdungsängste und materielle Barrikaden verdichten sich zu einer undurchdringlichen Textur der Grenze. ${ }^{2}$ In diesem Grenzraum sind allerdings nicht nur Mechanismen wirksam, die Migrant*innen verandern und sichtbar machen, sondern sie auch töten. In diesem Sinne spricht Nicholas de Genova im Eingangszitat vom Mittelmeer - dem tödlichsten aller europäischen Grenzräume - als einem Massengrab, das von der EU ausgehoben wird. Daher liegt der geopolitische Fokus des Beitrags auf dem Mittelmeer. Die tödliche VerAnderung vollzieht sich im mediterranen Grenzregime innerhalb eines militärisch-humanitären Komplexes, in dem, so die These, über Vergeschlechtlichung und Rassifizierung Migrant*innen mit spezifischen Affekten verkoppelt und dadurch de-/humanisiert werden. Durch diese Regierung der Anderen sollen regressive Migrationspolitik und ein repressives Grenzregime plausibilisiert werden.

Der Beitrag nimmt eine politiktheoretisch-dispositivanalytische Perspektive ein, um aufzuzeigen, wie der Grenzraum Europas im Sinne Michel Foucaults ein regierungstechnologisches „Ensemble“ darstellt, das „Diskurse, Institutionen, architekturale Einrichtungen, reglementierende Entscheidungen, Gesetze, administrative Maßnahmen, wissenschaftliche Aussagen, philosophische, moralische oder philanthropische Lehrsätze, kurz: Gesagtes

$1 \quad$ Verweisen möchte ich hier auf die Arbeiten der Grenzregimeforschung in einem europäischen Kontext, insbesondere auf Hess und Kasparek 2010; Heimeshoff et al. 2014; Hess et al. 2015 und Hess et al. 2017a.

Ich verzichte im Folgenden auf eine akkurate Unterscheidung zwischen der rechtlichen und politischen Organisationseinheit EU einerseits und dem soziokulturellen Imaginationsraum ,Europa ${ }^{6}$ andererseits, da diese Differenzierung eine klinische Exaktheit suggeriert, die in den gesellschaftlichen Diskussionen, narrativen Strukturen und (visuellen) Repräsentationen des Diskurses zu den Grenzen Europas nicht aufrechterhalten wird. 
ebenso wie Ungesagtes umfaßt [sic]“ (Foucault 1978, 119f.). Dieser Heterogenität des „Dispositivs“ der Grenze Rechnung tragend diskutiert der Beitrag die diskursive Produktion des Anderen, dessen affektiv-performative Stabilisierung sowie die materiell-technologische Kultur der Grenze. Damit lehnt sich der Beitrag an die Arbeiten Foucaults (2015 [1978f.]) zu Diskurs, Wahrheitsproduktion und Gouvernementalität an, wenngleich diese nicht im Mittelpunkt der Diskussion stehen. Die politische Theorie der Grenze speist sich in diesem Beitrag vielmehr aus Arbeiten, die sich an Foucault abarbeiten und seine Konzepte affekt- und geschlechtertheoretisch bzw. postkolonial fokussieren: Sara Ahmeds (2004) affekttheoretisch-diskursanalytische Arbeiten, Judith Butlers Theorie der Betrauerbarkeit (2006; 2016) und Achille Mbembes (2018) postkolonial angereichertes Konzept der Vergrenzung. ${ }^{3}$ Dadurch werden aus einer rassismus- und geschlechterkritischen Perspektive die trauerpolitischen Aspekte des europäischen Grenzregimes in den Blick genommen. Im Sinne der hier fokussierten drei Dimensionen des Dispositivs - Diskurs, Affekt/Performanz und Materialität - analysiert der Beitrag zunächst die Regierung der Anderen über ethnosexistische Affektökonomien, insbesondere in Rekurs auf visuelle Darstellungen des Ertrinkens des Anderen. Im Anschluss werden die diskursiv-performative sowie die technologische Dimension der Regierung der Anderen im europäischen Grenzregime erörtert und wiederum an das Motiv des Ertrinkens rückgebunden.

\section{„Wer hat Angst vorm schwarzen Mann?!“ Ethnosexistische Affektökonomien}

Am 2. September 2015 wurde der Leichnam eines Kleinkinds an die türkische Mittelmeerküste in der Nähe von Bodrum gespült. Das Foto des toten Jungen, rotes T-Shirt, blaue Hose, der Kopf auf die Seite geneigt, erregte weltweites Aufsehen. Wie bei anderen Fotos von leidenden, sterbenden oder toten Kindern haben sich die Trauer, die Wut, die Hilflosigkeit und das Unrecht in einer Momentaufnahme kondensiert, die den Menschen, die auf ihrer Fahrt über das Mittelmeer ertrinken, ein Gesicht und einen Namen gab: Aylan Kurdi. Die Fotojournalistin Nilüfer Demir schoss dieses Bild und berichtete später, sie „wollte den verstummten Schrei des Jungen hörbar machen" (Cigdem 2015). Doch in diesem Bild ist nicht nur das Individualschicksal eines Kleinkinds und seiner Familie repräsentiert. Vielmehr gibt

3 Alle Übersetzungen des Englischen und Französischen ins Deutsche stammen von der Autorin. 
das Bild den ungezählten und unsichtbaren Toten, die sich im Mittelmeer langsam zersetzen, die in Plastiksäcken in italienischen oder griechischen Polizeistationen und Auffanglagern verwesen, eine radikale, fast schon voyeuristische Sichtbarkeit.

Neben dem Namen des Jungen aus Syrien repräsentiert die Trope ,Lampedusa' das tägliche Sterben - im physischen wie im sozialen Sinne von Migrant*innen, die auf den Mittelmeerrouten und in Europa Gewalt erfahren: von den Schleuser*innen, den Versicherheitlichungsagenturen und migrationspolitischen Behörden. ${ }^{4}$ Jedoch stellt sich die Frage, warum gerade die italienische Mittelmeerinsel und der Name eines Kleinkinds zu Begriffen erhoben wurden, die das tägliche Sterben von ungezählten Geflüchteten auf ihrem Weg nach Europa auf einen Begriff fixieren bzw. auf ein einziges Bild bannen. Im Falle Lampedusas ist scheinbar Quantität entscheidend, denn dort sterben viele Menschen in regelmäßigen Abständen, die dann Sichtbarkeit erzielen, wenn sie an die Küsten der Insel geschwemmt oder von den Bewohner*innen - wortwörtlich - an Land gefischt werden. ,Lampedusa steht jedoch auch für eine idyllische Mittelmeerinsel, die eine Ambivalenz aufweist, die für die „südliche[.] Peripherie[.]“ (Soto Bermant 2017, 120) Europas charakteristisch ist: Sie versinnbildlicht zum einen die Geschichte des mediterranen Raums als Begegnungsstätte von Kulturen, Sprachen und Ethnien. Sie steht jedoch zum anderen für das Scheitern eines transkulturellen Europas. Erst der europäische Kolonialismus und sein aufklärungsphilosophisches Phantasma der Überlegenheit ziehen im mediterranen Raum strikte Trennlinien zwischen ,Europa', ,Arabien' und ,Afrika' und etablieren koloniale Muster, die das europäische Grenzregime nach wie vor heimsuchen. Europa ist von repressiven Politiken der sogenannten ,Kernstaaten und rassistischen Formationen ebenso unterwandert wie von (neo-)kolonialen Bedrohungsimaginationen, die den Mittelmeerraum vollkommen zu europäisieren trachten: ihn von ,afrikanischen', ,osmanischen', ,arabischen', ,maurischen', ,islamischen ${ }^{*}$ letztlich veranderten und ,nichtweißen ${ }^{6}$ - Residuen zu befreien. ${ }^{5}$

4 Zur Bedeutung von Lampedusa für das europäische Grenzregime verweise ich auf Heidrun Frieses ethnografische Studie von 2014. Zur Geschichte des Mittelmeerraums als Assemblage verschiedener Gesellschaften, Kulturen und Handelswege vgl. Laia Soto Bermant (2017, 126f., 132ff.) und ebenfalls Friese (2014, 114-130).

$5 \quad$ Im Folgenden werde ich weitestgehend mit dem binären Code ,weiß`,nichtweiß‘ operieren, um zu verdeutlichen, dass der rassifizierte Diskurs alle 


\section{Affektökonomien und das metonymische Gleiten des Diskurses}

,Aylan Kurdi‘ und ,Lampedusa‘ stellen Versuche dar, den Anderen - sein Sterben und gespenstisches Überleben - einzufangen, auf ein Bild zu bannen oder in einem Wort zu erfassen: ihn visuell, aber auch soziopolitisch und moralisch zu regieren. Die mediale Darstellung des Anderen ist dabei eng an die Produktion von Affekten gekoppelt. Mit Ahmed (2004) verstehe ich Affekte als produktiv, nicht possessiv: Sie werden über diskursive Operationen hergestellt, mit Bildern, Darstellungen und Begriffen verbunden und dadurch mit Bedeutung aufgeladen. Ahmed positioniert sich damit gegen psychosoziale Modelle, in denen Gefühle, quasi monadisch, aus sich selbst heraus entstehen und man diese einfach nur ,hat'. Diese Modelle depolitisieren Affekte, indem sie die Affektproduktion im isolierten Individuum verorten. Stattdessen sind Affekte immer auch gesellschaftlich-diskursiv eingebettet und somit politisch bzw. politisierbar: So werden z.B. in rezenten Migrationsdiskursen spezifisch affektiv aufgeladene (neo-)koloniale Rassifizierungen aufgerufen, um Politiken der Abschottung zu legitimieren.

Affekte werden in „affektiven Ökonomien“ (Ahmed 2004, 119) produziert. Das Modell der Affektproduktion ist an Marxens Warenzirkulationsmodell angelehnt und wird um Foucaults Überlegungen zur Wissensproduktion über diskursive und nichtdiskursive Praxen erweitert: Wissen wird über diskursive Operationen hergestellt, dann zirkuliert und amplifiziert. Dieses Wissen - auch non-propositionales oder schweigendes - wird über eine affektive Grundierung stabilisiert, indem Affekte mit bestimmten veranderten Figurationen, wie ,dem Migranten“, und Körpern „verklebt“ werden, um nor-

Leben, die als ,nichtweiß ${ }^{\prime}$ gelesen werden, undifferenziert ineinander kollabieren lässt. Damit reproduziere ich, einerseits, diese Binarität in einem gewissen Sinne, wenn auch kritisch gewendet; andererseits soll dies aufzeigen, wie gleichförmig ,Europa' auf veranderte Identitäten und Subjektpositionen blickt und diese zu einer scheinbar homogenen Masse amalgamiert. Ich verwende ,schwarz' statt ,nichtweiß', wenn der Diskurs diese Form der Rassifizierung wählt, um ,schwarz mit Dunkelheit, Düsternis, d.h. Bedrohung und Unheil, zu verbinden. Der Asteriskus betont die diskursive Konstruktion von vergeschlechtlichen Identitäten. Um neben der repressiven Dimension auch widerständige Formen des ,Nichtweißseins“ zu betonen, verweise ich auf $\mathrm{Ar}$ beiten zu Black Mediterranean. In Bezug auf Paul Gilroys Buch Black Atlantic von 1993 diskutiert eine wachsende Literatur aus Postkolonialer Theorie und Migrationsforschung unter dem Begriff des schwarzen Mittelmeers Phänomene und Logiken von Rassifizierung und (Neo-)Kolonialität; so z. B. Danewid 2017; Smythe 2018; Grimaldi 2019; Proglio et al. 2020. 
mative Rahmungen zu etablieren (Ahmed 2004, 119ff., 127, 130ff.). ${ }^{6}$ Jedoch ist die Verbindung zwischen Affekt und Figuration nicht stabil oder fixiert, sondern kann je nach Kontext unterschiedlich ausgedeutet und verschieden diskursiviert werden. Diese relationale Instabilität beschreibt Ahmed (2004, 118f.) mit dem Begriff des „metonymischen Gleitens“. Die Metonymie gehört sprachwissenschaftlich zu den Tropen: Sie ist, wie z. B. Metapher oder Synekdoche, eine rhetorische Stilfigur, bei der ein sprachlicher Ausdruck nicht in der wörtlichen Bedeutung, sondern in einem übertragenen Sinne Verwendung findet. Klassischerweise stehen der eigentliche und der ersetzte Ausdruck in einer nahen sachlichen Beziehung zueinander. Im Konzept des metonymischen Gleitens ist diese Nähe jedoch kontingent, wird erst diskursiv hergestellt und dabei mit Affekten versehen. In diesem Sinne gehe ich davon aus, dass der europäische Migrationsdiskurs in der tropischen Figuration des männlich gelesenen Migranten eine metonymische Nähe zwischen den Begriffen des Todes und der Bedrohung herstellt, diese mit verschiedenen Affekten belegt und sich daher zwischen beiden Begriffen hin- und herbewegt - also: gleitet. Die Figuration Migrant wird je nach Sprechposition und diskursiver Sinnproduktion mit Bedrohung und/oder mit Tod assoziiert und setzt spezifische Affektökonomien in Gang. Da sich in affektiven Ökonomien Machtverhältnisse manifestieren und dort auch stabilisiert werden, kommt in ihnen immer auch epistemische Gewalt zum Tragen. ${ }^{7}$

Auf mein Beispiel übertragen, ist zu beobachten, dass das Bild Aylan Kurdis und seine Zirkulation zu einer bestimmten Produktion von Affekten beitragen: Weil dort ein Kind liegt, das mit Unschuld und Reinheit assoziiert scheint, wird Mitleid generiert, das vor allem humanitär-moralisch konno-

6 Ahmed (2004) verwendet im Englischen „sticky“ und „stickiness“ (120, 122, $127,130)$ sowie „adherence“ $(119,133)$, um einerseits zu verdeutlichen, wie bestimmte Bedeutungen an Worten kleben, indem zwischen ihnen eine Verbindung diskursiv hergestellt und mit Bedeutungen versehen wird. Ahmed verweist aber auch auf „sticking together“ im Sinne von „zusammenhalten“ und „zueinanderstehen“ in Bezug auf politische und gesellschaftliche Zugehörigkeiten und Kollektivbildung (,adherence as coherence“, 133).

7 Ahmed entwickelte das Modell der affektiven Ökonomie auch vor dem Hintergrund des US-amerikanischen war on terror nach 9/11, der einen Nexus zwischen Terrorismus, (antimuslimischem) Rassismus und Migration etabliert, der, laut Ahmed (2004, 119ff.), den Affekt „Hass“ hervorgebracht hat, um Migrationspolitik mit sicherheitspolitischen Erwägungen im Kampf gegen (islamistischen) Terrorismus verbinden zu können. 
tiert ist. Darüber hinaus wurde die Hautfarbe Aylan Kurdis in den Diskussionen und medialen Repräsentation zwar nicht als ,weiß` gelesen, allerdings galt sie als hell genug, um als noch ,europäisch' gelesen werden zu können. Weiterhin stammten Aylan und seine Familie aus Syrien, dessen Bürgerkrieg durch seine Grausamkeit - in Form hoher ziviler Opferzahlen - und die Diktatur Baschar al-Assads seit Jahren hohe mediale Aufmerksamkeit in Europa erfährt. Syrische Migrant*innen gelten als schutzbedürftige „Flüchtlinge“, deren erzwungene Migration aus Syrien unter den humanitären Schutz der Genfer Flüchtlingskonvention fällt - und damit legitim wird. Dies trägt zur Produktion einer moralisierenden Affektökonomie bei, die vor allem Mitleid erzeugt. Damit wird das Ertrinken Aylan Kurdis primär moralisch bearbeitet und konnte sich dadurch auch rasant verbreiten. Über Mitleid evoziert es einen weltweiten moralischen Aufschrei - denn: „[s]olche Bilder brennen sich in die Netzhaut ein“, kommentiert der Medienethiker Alexander Filipovic in einer moralisierenden Geste: „Ich hatte Tränen in den Augen, und die Tränen sind auch gelaufen. Und sich vorzustellen, dass dieser Junge das eigene Kind wäre - kaum auszuhalten“ (zit. in Maxwill 2015).

Nicht nur vermag die Repräsentation des migrantischen Todes, noch genauer: des toten veranderten Kindes, eine komplexe Situation in einem Bild abzubilden. Durch seine stete Wiederholung in verschiedenen Berichterstattungen, durch diskursive Praxen und künstlerische Reproduktionen wurde das Foto von Aylan Kurdi inzwischen ikonisiert (Oberprantacher 2018, 212). Dabei richten sich die medialen Darstellungen des toten Kindes vorwiegend an die affektive Betroffenheit einer ,westlichen“ „humanitarian viewership“ (Athanasiou und Sheikh 2019, 96), durch deren hegemoniale Position innerhalb globaler normativer Ordnungen sich dann auch der ,Impact-Faktor ' des Bildes erhöht. Eine Politisierung der Ursachen und strukturellen Missstände, die zum Sterben von Personen auf dem Mittelmeer führen, wird dabei zugunsten einer moralisierend-humanitären Diskursivierung nahezu ausgeblendet. Denn die Moralisierung verfügt über hohe Affektivität, weshalb die Anschlusskommunikation gewährleistet ist. Daher sind hinter der massiven medialen Zirkulation des Bildes nicht nur moralisch-humanitäre Erwägungen zu vermuten, sondern auch ein wirtschaftliches Verwertungsinteresse, das über affektive Ökonomien (!) aufrechterhalten wird. 


\section{Zwischen Bedrohung und Tod: Ethnosexistische Repräsentationen des Anderen}

Dass die metonymische Relation zwischen der Figuration ,totes Kind' und der des ,Migranten“ in andere diskursiv-affektive Kontexte gleiten kann, zeigt eine Karikatur, in der eine Skizze der ikonografischen Fotografie des toten Aylan Kurdi rekontextualisiert wird. Kurz nach der Silvesternacht 2015/16 veröffentlicht das Satiremagazin Charlie Hebdo eine Karikatur von Laurent Sourisseau, auf der man zwei Männer sieht, die mit hervortretenden, lüsternen Augen und weit nach vorne gestreckten Armen zwei schreiende, panische Frauen jagen. Durch einen ausgeprägten Unterkiefer und überproportional lange Arme weisen die Männer Auffälligkeiten zur rassistisch-animalistischen Darstellung von ,schwarzen Männern' als Affen auf. ${ }^{8}$ Über dem Bild sieht man, graphisch hervorgehoben, das Wort migrants, darunter auf der linken Seite einen Kreis, in dem eine Zeichnung des Fotos von Aylan Kurdi zu sehen ist; daneben steht: „Was wäre aus dem kleinen Aylan geworden, wenn er groß geworden wäre?" Die Antwort erfolgt am unteren Rand der Karikatur: „Arschgrapscher in Deutschland“. In der Karikatur wird dargestellt, dass bereits in ,Aylan“ das Animalische, Hypersexuelle und Primitive des Anderen schlummerte. Dieses imaginierte Bedrohungspotential wäre ,Wirklichkeit' geworden, falls er ein gewisses Alter erreicht hätte. Hier produziert der Diskurs über die Differenzkategorien Geschlecht, ${ }^{9}$ race und Alter Affektökonomien der Angst, der Furcht und des Hasses, die über bestehende (neo-)koloniale Stereotype ebenso bestärkt werden wie über antimuslimische Ressentiments.

8 Auf die tierethischen und zoopolitischen Implikationen einer Verunglimpfung von Personengruppen durch den Vergleich zu Tieren kann ich an dieser Stelle nur verweisen. Tiere zunächst abzuwerten (,es sind ja nur Tiere ...'), um im Anschluss bestimmte Personengruppen zu animalisieren, d.h. zu dehumanisieren, um ihre Minderwertigkeit hervorzuheben, ihre Tötung und Schlachtung zu legitimieren, ist ein Motiv, das sich durch die „Zornpolitik“ europäischer Diskurse zu Alterität zieht; vgl. dazu die emotionshistorische Untersuchung von Uffa Jensen (2017), die die Prozesse der Dehumanisierung u. a. für antisemitische Ressentiments darstellt.

9 Zu einer weiterführenden Diskussion der notwendigen Integration von geschlechter- und rassismuskritischen Ansätzen in Migrations- und Grenzregimeforschung s. ebenfalls Neuhauser et al. 2017. 
An dieser Karikatur wird deutlich, wie im europäischen Grenzregime der migrantische Tod mit der Bedrohung durch männlich markierte und vermeintlich muslimische Migranten diskursiv zum Gleiten gebracht wird. Als Resultat konnte beobachtet werden, wie es nach ,Köln` zu einer problematischen Verflechtung von (antimuslimischem) Rassismus und einem „westlichen Feminismus“ (Mohanty 1984, 333f.) kam, den Sabine Hark und Paula-Irene Villa (2018, 40, 68f., 78) als „toxisch“ bezeichnen. Denn hier werden geschlechterkritische und feministische Debatten mit rassistisch grundierten Diskussionen verzahnt, die zu Allianzen zwischen Rassismus und - einem semantischen - ,Feminismus führen. Dabei unterliegt diesem Diskursfragment keine feministische Perspektive, wenn man diese als kritische Hinterfragung von Geschlechterverhältnissen als Machtverhältnissen versteht, denn als essentialisierende Bewegung zur Befreiung von ,Frauen“ aus patriarchalen Strukturen. Dass der Diskurs zunächst ausschließlich binäre Geschlechterkodierungen zulässt und dann sowohl männliche als auch weiblich markierte Andere Sexismus in unterschiedlichen Manifestationen erfahren, bringt Gabriele Dietze $(2016,178)$ im Begriff des „Ethnosexismus“ auf den Punkt, der

Sexismen [beschreibt, MG], denen sexualisierte Rassismen zugrunde liegen, und die gegenüber Frauen und Männern aus ethnisch, religiös und deshalb meistens auch sozial marginalisierten Gruppen wirksam werden [...]. Ethnosexismus wird hier als eine Art von Kulturalisierung von Geschlecht verstanden, die ethnisch Markierte aufgrund ihrer Position in einer angeblich problematischen oder, rückständigen' Sexualität oder Sexualordnung diskriminiert. ${ }^{10}$

Dietze $(2016,178)$ zeigt auf, wie eine „sexualpolitisch argumentierende Migrationsfeindlichkeit“ völkisch-nationalistische Grenzziehungen stabili-

10 Das Echo dieser ethnosexistischen Vorstellung hallt in einem beliebten Spiel meiner Kindertage nach: „Wer hat Angst vorm schwarzen Mann?!“ - eine Figur, die Furcht und Panik auslöst, da sie versucht, einen einzufangen und letztlich ,zu töten‘: Wenn der ,schwarze Mann' einen gefangen hatte, durfte man nicht mehr am Spiel teilnehmen. Darauf rekurriert die Kapitelüberschrift, womit auch gezeigt werden soll, wie tief Rassismus in die gesellschaftliche Alltagsstruktur einwandern kann. Historisch geht das Spiel vermutlich auf den „schwarzen Tod“ - die Pest - zurück, zeigt aber dadurch deutlich auf, wie ,schwarz' (emotions)historisch mit Krankheit, Bedrohung und Tod verwoben ist. Dies spiegelt sich dann in der rassifizierten Semantik des Anderen als ,schwarz' wider. 
siert, indem die Intersektionen von Geschlecht, Ethnie, Sexualität, Religion, Klasse/Milieu - und ich möchte Alter ${ }^{11}$ hinzufügen - mit einer geopolitischen Positionierung verschränkt werden. D.h. dass für den Wissens- und Machtkomplex Migration/Tod des europäischen Diskurses verschiedene Affekte aufgerufen werden, die je nach Betonung von Differenzkategorien unterschiedlich ausfallen (Mecheril und van der Haagen-Wulff 2016, 125; Hark und Villa 2018, 82f.). In der patriarchal-kolonialen Struktur Europas werden diese Differenzkategorien - insbesondere in Bezug auf Geschlechteridentitäten und Alter der Migrant*innen - zwar diskursiv immer wieder neu ausgehandelt und affektiv umgedeutet, halten sich jedoch erstaunlich stabil an die Konstruktion vermeintlich klarer, fixierter Darstellungen von alterisierten Migrant*innen: Zum einen werden weiblich gelesene Migrantinnen vorwiegend in Viktimisierungsoperationen eingebettet. Der ,hilfsbedürftigen und ,unschuldig in Not geratenen Migrantin' sei durch die Aufnahme in die europäische Gesellschaft zu helfen, damit sie die geschlechter- und sexualpolitisch ,rückständigen' Herkunftsländer und -regionen hinter sich lassen kann. Die geschlechter- und gleichstellungspolitischen Schieflagen und Machtasymmetrien der sog. Aufnahmeländer bleiben hier frappierend unreflektiert. Die Figuration ,Migrantin' wird mit explizitem Verweis auf ihre Feminität so gedeutet, dass der Diskurs metonymisch zwischen den essentialisierenden Ontologien ,Frau', ,Opfer' und ,Migrantin' gleitet. Europa, mythologisch-etymologisch feminisiert, tritt hier als ,solidarische' Retterin auf. Denn die in der misogynen Kultur ihres Heimatlandes unterdrückte ,Schwester' kann durch die vermeintlich progressive Geschlechterpolitik ,Europas' gerettet werden. In einer Reiteration von Gayatri Chakravorty Spivaks $(1988,92)$ berühmt gewordenem Bonmot - „White men saving brown women from brown men" - kann also die ,nichtweiße Frau“ von der ,weißen Frau' Europa gerettet werden, da beide durch ,nichtweiße Männer' bedroht sind. ${ }^{12}$ In der nach wie vor patriarchal operierenden Gesellschaft Europas

$11 \quad$ So wurden über die Kanäle rechtskonservativer und rechtsradikaler Parteien und Bewegungen immer wieder Gerüchte zirkuliert, nach denen sich viele alleinstehende - meist männlich gelesene - Geflüchtete als minderjährig ausgeben, ,in Wahrheit jedoch viel älter seien. Auch die Versicherheitlichungstechnologien des europäischen Grenzregimes unterstützen diese Diffamierungen, wenn über medizinische Tests das Alter von Geflüchteten überprüft wird, wie dies z. B. in Dänemark gängige Praxis ist (Schwennicke 2018).

$12 \mathrm{Zu}$ dieser Logik eines, westlichen“ oder, weißen' Feminismus verweise ich auf die Beiträge „Was geschah wirklich an Silvester?" und „Silvester: Geht Ideolo- 
kann diese Solidarität aber nur bestehen, solange die grundlegende Hegemonie des Männlichen nicht hinterfragt wird.

Zum anderen sind als männlich gelesene Migranten dagegen vorwiegend in Dämonisierungsoperationen eingebettet. So gelten als ,muslimisch', ,arabisch` oder ,schwarz' markierte erwachsene Männer als bedrohlich für die vermeintlich fortgeschrittene ,Zivilisation“ Europas. Nur wenn sie jung genug sind, um die entscheidende formative Phase in Europa zu durchlaufen, können sie in die angeblich fortschrittliche Sexualordnung Europas ,erfolgreich' eingepasst werden - aber selbst dies wird dem Aylan der Karikatur verwehrt. Die als ,nichtweiß', d. h. ,uneuropäisch' markierten Männer stellen in den rassifizierten Fantasien Europas immer schon eine Bedrohung seiner normativen (Sexual-)Ordnung dar. Die animalische ,Natur' des hypersexualisierten Anderen kann nicht gezähmt werden; selbst dann nicht, wenn er in Europa aufwächst. „Das Dämonisierungs-Narrativ [...] des ,schwarzen Mannes', vor dem sich vor allem frau in Acht zu nehmen habe", so Hark und Villa $(2018,64)$ pointiert, „amalgamiert bildlich zwei Differenzen, die sich dabei wechselseitig essentialisieren: schwarz/weiß und männlich/weiblich." Auch in den medialen Darstellungen und gesellschaftlichen Diskussionen, die sich um das europäische Grenzregime ranken, wirkt diese Dualität nach: In ihnen wird eine „primitive, weniger zivilisierte, durch Sozialisation einverleibte kulturelle Ontologie einer patriarchalen Misogynie“ (Hark und Villa 2018, 64) inszeniert, die den virilen ,schwarzen Mann` hypersexualisiert (Dietze 2016). Er wird der ,Keuschheit' der ,weißen Frau' entgegengesetzt, die von der ungezügelten Sexualität des ,schwarzen Mannes` zu beschützen sei. ${ }^{13}$

Trotz dieser Unterschiede zwischen weiblich und männlich gelesenen Migrant*innen, kann mit Hark und Villa $(2018,29)$ geschlussfolgert werden, dass Migrant*innen in eine „herrschaftliche Logik der (De-)Humanisierung“

gie vor Realität?" von Alice Schwarzer von den Jahresanfängen 2016 und 2017 in EMMA, einer deutschen feministischen Zeitschrift, die von Schwarzer 1977 gegründet wurde und der sie als Chefredakteurin vorsteht.

13 Hier wirken misogyne Stereotype, die auch ,die europäische Frau' als hilflos, unschuldig und wehrlos imaginieren bzw. sie vor ,unreinen' (sexuellen) Beziehungen zum männlich gelesenen Anderen warnen. Die Diffamierung und das Verbot von sexuellen Beziehungen zwischen ,weißen Frauen' und ,schwarzen Männern' spiegelt sich auch historisch-rechtlich in den Slave Codes und dem Code Noir wider: Bei Aufdeckung dieser Beziehungen wurden Frauen meist sozial geächtet, Männer wurden mit großer Wahrscheinlichkeit hingerichtet (Därmann 2020, 19-23). 
eingebunden werden: Weiblich gelesene Migrantinnen werden humanisiert, was ihnen zwar ihre Existenz als Frau - im Sinne ihres "nackten Lebens“ (Agamben 2002, 18) - lässt, ihnen darüber hinaus jedoch nichts anderes zugesteht. Damit ist die Migrantin radikal entpolitisiert und Adressatin humanitärer Interventionen seitens Europas. Im Gegensatz dazu werden männlich gelesene Migranten dehumanisiert, damit die Dämonisierungsund Animalisierungsnarrative e/affektiv greifen können. Die als männlich, erwachsen und ,nichtweiß markierten Körper evozieren rassifizierte Fantasien statt humanitäres Mitgefühl. Sie erwecken die Vorstellung des bedrohlichen ,schwarzen Mannes', die tief in die (neo-)kolonialen Imaginationen Europas eingeschrieben ist (Fanon 1985 [1952], 24ff., 66ff.; Said 1979, 1-8).

Diese herrschaftlich-koloniale Logik zeigt sich kaum deutlicher als in den medialen Darstellungen des ,überfüllten Flüchtlingsbootes', um noch einmal auf ,Lampedusa' zu sprechen zu kommen, in dem sich die bildhafte Steigerung der Dämonisierung der ,schwarzen Masse' manifestiert:

Diese Repräsentationen rahmen und iterieren die herrschenden, rassistisch konnotierten politischen Metaphern von einem Ansturm biblischen Ausmaßes, von schwarzen Migrantenströmen oder von humanitärer Katastrophe und armen Opfern sinisterer Menschenhändler, die durch effizienten Grenzschutz und ,Grenzmanagement" eingedämmt und unter Kontrolle gehalten werden müssen. (Friese 2014, 185)

Zwar gibt es unzählige mediale Darstellungen, die zeigen, wie diese Boote umkippen, Menschen aus ihnen herausfallen und zu ertrinken drohen, doch in den europäischen Leitmedien wird das ,Boot voller schwarzer Männerkörper' vor allem in Bedrohungsoperationen verwoben und mit Affekten von Angst und Furcht verklebt. Hier tritt auch eine unheimliche Verbindung das metonymische Gleiten - zwischen der Figuration des Bootes als ein, volles Boot' in Sinne einer antimigrationspolitischen Trope und als ein ,überfülltes Flüchtlingsboot' zu Tage, das in den medialen und gesellschaftlichen Repräsentationen des militärisch-humanitären Komplexes des EU-Grenzregimes zwischen Mitleid und Empörung sowie Überforderung und rassifizierter Angst changiert. ${ }^{14}$

14 Selten sieht die europäische Zuschauer*innenschaft diese Boote in ihrem inneren Aufbau. In den Nachrichten, Zeitungen und im Internet werden Videoclips und Bilder gezeigt und geteilt, in denen man meist von der Seite gefilmte Boote, manchmal von oben, sehen kann. Man sieht eine Vielzahl von ,nicht- 


\section{Die „tödlichst[e] (See-)Fluchtroute der Erde“: Die diskursiv- performative Produktion von Nicht-/Betrauerbarkeit}

Im Gegensatz zur migrationsfeindlichen Trope des ,überfüllten Bootes s sind die Menschen auf dem übervollen ,Flüchtlingsboot' in ihrer physischen Existenz bedroht: Auch der humanitär-militärische Komplex erkennt dies an, wenn der UNHCR (2019) berichtet, dass in den ersten Wochen des Jahres 2019185 Menschen im Mittelmeer ertrunken sind, weil durch „die zurückgefahrene Seenotrettung [...] die Flucht über das Mittelmeer wieder zur tödlichsten (See-)Fluchtroute der Erde geworden [ist]“. Das Mittelmeer steigt damit zum „veritablen Epizentrum [...] tödlicher Grenzüberquerungen“ (De Genova 2017, 3) auf. Die Toten werden hier zwar gezählt und statistisch erfasst, doch mit Butler (2016, xx) bleibt zu fragen, ob diese Toten auch zählen?

Butlers (2006; 2015; 2016 [2009]) trauerpolitische Arbeiten zerlegen die Logiken öffentlicher Affektproduktion und fragen und insbesondere nach den diskursiven Mechanismen einer beobachtbaren differenten Distribution von Trauer. Dabei rückt Butler (2015, 40f.) den Begriff des Rahmens (frame) in den Mittelpunkt, der darüber entscheidet, wer ,gesehen, gehört und anerkannt“ werden kann. Rahmen werden über soziale, kulturelle und politische Diskurse - auch auf einer schweigenden Ebene im Sinne von Affekten und Performativität - hergestellt und eingeübt, wodurch eine „visuelle und diskursive Normalisierung“ (Butler 2016, xvii) in Gang gesetzt wird, die das Sagbare vom Unsagbaren, das Sichtbare vom Unsichtbaren und das Intelligible vom Nichtlesbaren differenziert. Rahmen regulieren die sinnliche und affektive Wahrnehmung und steuern schließlich Handlungen. Sie

weißen' Männerkörpern, die oft nur diffus als Einzelne zu erkennen sind - was man nicht sieht: Meist bestehen nur die äußeren zwei, drei Reihen aus Männern. Zu ihrer eigenen Sicherheit - vor dem Kentern, vor der Sonne, vor unerwünschten körperlichen Übergriffen (!) - sitzen Frauen, Kranke und Kinder in der Mitte des Bootes. Diese räumliche Verteilung innerhalb des Bootes ist eine gängige Praxis, die immer wieder von Seenotrettungsorganisationen wie Sea Watch oder Sea Eye dokumentiert und kommuniziert wird. So z. B. von Michael Schwickart, Head of Fundraising und Crewmember von Sea Watch, in einem Vortrag am 18. Dezember 2018 an der FAU Erlangen-Nürnberg im Rahmen der Veranstaltungsreihe „Safe Passage - Safe Harbours? Debatten um die neue EU-Migrationspolitik“. Aus geschlechterkritischer Perspektive sei darauf hinzuweisen, dass diese traditionelle Aufteilung des Bootes zu problematisieren ist, da sie die Reproduktion patriarchaler Strukturen zumindest suggeriert. 
haben eine normalisierende und damit auch normierende Funktion und können daher als eine spezifische Form der Grenzziehung und Sichtbarmachung verstanden werden..$^{15}$

Auch Grenzpolitiken und Migrationsdiskurse operieren über frames: Sie ziehen durch rechtliche Rahmungen einerseits territoriale Grenzen; doch sie definieren dadurch andererseits Zugehörigkeit, sodass sich die juridico-politischen Rahmen in soziomentalen Demarkationen widerspiegeln - und vice versa. Weiterhin legen sie damit die (affektiven) Normen der Nicht-/Betrauerbarkeit von Grenztoten fest und erlangen dadurch eine normative Dimension (Butler 2006, 46). Gesellschaften bestimmen über diskursive Praxen, wer wann wie und weshalb (nicht) öffentlich betrauert werden kann. Recht, Politik und Moral verstärken diese gesellschaftlichen Schließungstendenzen, die sich in Migrationspolitiken ebenso manifestieren wie in Grenzregimen. In diesem Sinne argumentiert Butler (2016, xix), dass Rahmungen dazu führen, die „radikale Nicht-Betrauerbarkeit von bestimmten Populationen" zu akzeptieren. Gesellschaften produzieren normalisierende Rahmungen, stabilisieren diese über sprachliche und implizite, d.h. affektiv-performative Verständigungen, um letztlich die „unterschiedliche Distribution von Betrauerbarkeit“ (Butler 2016, xix) aufrechterhalten zu können.

Betrauerbarkeit definiert Butler vor allem über eine alteritätspolitische Bewegung der Negation, die diskursiv hergestellt und reiterativ-performativ stabilisiert wird:

Ungrievable lives are those that cannot be lost, and cannot be destroyed, because they already inhabit a lost and destroyed zone; that are, ontologically, and from the start, already lost and destroyed, which

15 Wie bei Ahmed werden Butlers (2006; 2016 [2009]) Arbeiten zu Betrauerbarkeit durch die Attentate am 11. September 2001 initiiert. Butler seziert die verschiedenen Machtverhältnisse, die sich während der öffentlichen Trauerfeiern und des anschließenden war on terror manifestieren; und argumentiert, dass bestimmte rassifizierte Populationen kaum oder gar nicht in die öffentliche Trauer und ihre Politiken integriert wurden. Trotz dieses anderen Kontextes, in dem Butler das Konzept der Betrauerbarkeit entwickelte, wird es seit mehreren Jahren in der Grenzregimeforschung und den Border Studies produktiv verwendet; so widmet Social Research seine Ausgabe vom Sommer 2016 der Thematik „Borders and Politics of Mourning“ (hrsg. von Alexandra Délano Alonso und Benjamin Nienass), in dessen Beiträgen Butlers Konzept immer wieder Anwendung findet. 
means that when they are destroyed [...], nothing is destroyed. (Butler 2016, xix)

Nichtbetrauerbare Leben werden also in ihrer Zerstörung, in ihrem Tod, nicht perzipiert, da sie aus den Rahmen der Betrauerbarkeit herausfallen - bis zu dem Punkt, dass ihr Tod nicht wahrgenommen wird, weil ihr Dasein gar nicht als Leben gelesen wurde. In einem melancholisch-zynischen Anachronismus zählt der Tod des Anderen nicht, weil er nie gelebt hat: nie als Leben zählte.

Die Toten des europäischen Grenzregimes werden also nicht oder nur unter bestimmten Bedingungen - wie z. B. im Falle Aylan Kurdis - betrauert, weil ihr Tod nicht intelligibel ist: weil sie gar nicht gestorben sind, weil sie gar nicht als lebend wahrgenommen wurden. ${ }^{16} \mathrm{Um}$ auf mein Beispiel zurückzukommen: Solange Aylan Kurdi als ,Kind‘ diskursiviert wird, sind Mitleid und Wut trauerpolitisch garantiert, während er als ,heranwachsender Migrant' als nichtbetrauerbar konstruiert wird. Dieses diskursiv-metonymische Gleiten verstärkt sich durch die Integration der Differenzkategorie race. Denn in die Konstruktion von ungrievable lives ist ein „rassifiziertes Imaginäres“ eingeschrieben, das als ,arabisch“ bzw. ,muslimisch“ markierte Andere in einem Anflug von „racial hysteria“ pauschalisierend diffamiert und schließlich dämonisiert (Butler 2006, 32, 39). Insbesondere in seinen ethnonationalistischen und neokolonialen Anrufungen des Autoritären operiert das europäische Grenzregime mit dieser „Dämonisierung des Anderen“ (Castro Varela und Mecheril 2016), um regressiv-abschottende Migrationspolitik und hermetische Grenzziehungen zu legitimieren.

Mit dem Konzept der Betrauerbarkeit kann daher analysiert werden, wie migrantische Leben durch diskursive Operationen verschiedener Akteur*innen des europäischen Grenzregimes als nicht-/betrauerbar konstruiert werden. Dabei bewegt sich dieser Umgang zwischen einer humanitär-neokolonialen Willkommenskultur, die auf eurozentrischen Moralismen basiert, einer neoliberalen Managementstruktur, die von Optimierungs- und

16 Ich verweise hier exemplarisch auf die Aktionskunst Die Toten kommen im Juni 2015 des Zentrums für Politische Schönheit (ZPS), die kritisch auf die Konstruktion nichtbetrauerbarer Leben verweist. Eine umfassende Dokumentation der verschiedenen Performances dieser politisch-künstlerischen Aktion findet sich auf der Seite des ZPS (https://politicalbeauty.de/toten. html). Zu einer Kritik am ZPS aus trauer- und alteritätspolitischer Perspektive: vgl. von Bieberstein und Evren 2016. 
Selbstverantwortungsvorstellungen geprägt ist, und einer ethnonationalistischen Anrufung des Eigenen, die von ,völkischer Einheit und ethnischer Reinheit schwadroniert (Hess et al. 2017a; Kersting und Leuoth 2020). Diese drei Elemente des europäischen Grenzregimes spiegeln sich auch in der materiellen Kultur der Grenze wider, durch die veranderte Personengruppen regiert werden. Diese verschiedenen Diskurselemente verzahnen sich in einem Komplex, den ich als humanitär-militärisch beschreibe. Er bringt verschiedene Formen von Betrauerbarkeit und Sichtbarkeit hervor: In den materiellen Manifestationen des Grenzregimes, wie der Zaunanlage, sowie in seiner technologischen Versicherheitlichung wird Betrauerbarkeit hergestellt, die insbesondere durch Sichtbarmachungsrahmen reguliert wird. Dabei hat Sichtbarkeit immer auch mit Verräumlichung und Materialisation zu tun, die den unsichtbaren Diskursoperationen Gestalt verleihen und sie begreifbar machen.

\section{„Around the year 2000, it was just a normal fence“. Regierungstechnologien der Sichtbarmachung}

In einem Artikel zu „Sicherheit und Migration“ im Juli 2018 beschreibt Achille Mbembe für den sicherheits- und migrationspolitischen Konnex das Phänomen der „großen Vergrenzung“ - einen „Prozess, durch den Weltmächte permanent bestimmte Räume in Orte verwandeln, die für bestimmte Gruppen von Menschen undurchquerbar sind“. Damit verweist er auf die sukzessive räumliche Ausdehnung der Grenze, die unter spätkapitalistischen Bedingungen keine klare Linie mehr darstellt, sondern einen Raum. Hiermit verweist Mbembe auf die zentrale spatialtheoretische Differenzierung zwischen „Räumen“ und „Orten“: Während Orte und die in ihnen wirkende Ortung auf eine gewisse Ordnung - im Sinne Carl Schmitts (1974 [1950], 13-21) -, d.h. Fixiertheit, Normierung und Definiertheit, verweisen, kann Raum als eine unabgeschlossene Topographie des Politischen verstanden werden, die in einem Arendt'schen Sinne Pluralität, Alterität und Freiheit ermöglicht (Gebhardt 2014, 48-61). Schließlich macht Mbembe, auch im Anschluss an seine Arbeiten zu „Nekropolitik“ (2003 ${ }^{17}$ ), auf das Phänomen der Nicht-/Betrauerbarkeit aufmerksam: Im Prozess der Vergrenzung geht es auch „um die bewusste Vermehrung der Orte des Verlusts und der Trauer, an denen die Leben junger Menschen, die man als unerwünscht einge-

$17 \mathrm{Zu}$ einer detaillierten nekropolitischen Perspektivierung des europäischen Migrationsregimes s. Gebhardt 2020. 
stuft hat, zerstört werden“ (Mbembe 2018). Denn an den Grenzen und in den Grenzräumen Europas sterben Menschen, die nicht betrauert werden können, da sie nicht als Leben gezählt haben. Im Umkehrschluss können Familie und Freund*innen nicht um diese ungezählten Leben trauern, da ihr Sterben nicht dokumentiert wurde. Einer dieser tödlichen Grenzräume Europas ist das Mittelmeer. In ihm kommen Technologien der Versicherheitlichung zum Einsatz, die seine Tödlichkeit einerseits verstärken, jedoch auch aufzuheben suchen. Ich verstehe Technologie zweifach: Zum einen als die militärisch-sicherheitspolitische Aufrüstung der Grenze durch Technik. Zum anderen jedoch auch als Subjektivierungstechnik des Grenzregimes, durch die veranderte Personengruppen als nicht-/betrauerbar regiert und dadurch in ihrer Alterität radikal un-/sichtbar werden.

\section{Technologien der Vergrenzung}

Grenzräume bringen nicht nur „Repression“, sondern immer auch umkämpfte und widerständige „Zwischenräume“ hervor (Dudziak und Volpp 2006, 3). Ganz im Sinne der spatialtheoretischen Differenzierung sind Räume nicht nur Orte der Ordnung und Ortung, die repressive Strukturen (re-)produzieren. Vielmehr entstehen in ihnen auch widerständige Subjekte und Strukturen. Betrachten wir zunächst die repressive Dimension, wird deutlich, dass die sukzessiv erweiterte europäische Außengrenze zu einem Grenzraum über Recht etabliert, z. B. in den zunehmenden Extraterritorialisierungsbestrebungen von Lagerstrukturen nach Nordafrika, und ,illegale Migration damit überhaupt erst produziert wird. Durch die räumliche Ausweitung der Grenze wird diese diffus, denn sie ist keine klar markierte Linie mehr. Die Grenze wird damit einerseits unübersichtlicher und weniger greifbar und muss daher, andererseits, stärker bewacht werden. Die Grenzkontrolltechnologien und ihre administrativen Institutionen wirken ein diffiziles und engmaschiges Gewebe des Grenzraums und verdichten ihn zu einer undurchdringlichen Textur. Noch genauer muss man argumentieren, dass durch die juridico-politische Produktion von räumlich ausgedehnten Grenzanlagen gerade erst derjenige unübersichtliche Grenzraum erschaffen wird, den es dann zu schützen, zu bewachen und abzusichern gilt. Die juridico-territoriale Ausdehnung der Grenze zu einem Raum durch rezente Migrationspolitiken und Grenztechnologien evoziert also die Notwendigkeit, den Grenzraum militärisch-versicherheitlichend aufzurüsten.

So ist der mediterrane Grenzraum zunehmend mit Technologien der Sichtbarmachung, Identifikation und Überwachung überfrachtet, z. B. durch 
medizintechnische Verfeinerungen zur Ortung von Herztönen, wie sie auf Schiffen der Grenzschutzagentur FRONTEX zu finden sind. Hier greifen Ordnung und Ortung, wie oben erörtert, ineinander: Rhetorisch wird die Ortungstechnologie als Instrument im Kampf gegen den Menschenhandel ausgegeben..$^{18}$ Jedoch dient sie vor allem einer radikalen Sichtbarmachung illegalisierter Migrant*innen und einer zunehmenden Kriminalisierung von Migrationsbewegungen. Damit sind wissenschaftliche Erfassungstechniken zunehmend „integrale[r] Bestandteil des Grenzregimes und seiner Ökonomie“, schreibt Friese $(2014,190)$ und führt aus, dass „Statistiken, Tabellen und Kartierungen [...] Teil der Sicherheitstechniken, der Gouvernementalität und ihrer Weltvermessung" sind. Friese verweist beispielhaft auf das International Centre for Migration Policy Development (ICMPD), das in der neoliberalen Rhetorik des Migrationsmanagements von 2011 bis 2014 die Interactive Map on Migration (i-map) einsetzte, um Daten zu migratorischen „Strömen“ und „Knotenpunkten“ zu sammeln. Als Ziel wird auch hier „combatting migrant smuggling“ durch grenzüberschreitende Kooperation formuliert (ICMPD); das ICMPD arbeitet u. a. mit Europol, FRONTEX, Interpol und dem UNHCR zusammen - einem Verbund, der paradigmatisch für einen militärisch-humanitären Komplex des Migrationsmanagements steht, wie er typisch für das europäische Grenzregime ist.

Doch auch systemkritische Projekte, die auf das Sterben im Mittelmeer aufmerksam machen, bedienen sich fortgeschrittener Überwachungstechnologien. Sie tragen letztlich auch zu einer Datengenerierung bei, die durch repressive Technologien und institutionelle Komplexe für eine restriktive Migrations- und Grenzpolitik instrumentalisiert werden kann. So überwacht (!) die zivilgesellschaftliche Organisation WatchtheMed als „civilian watchtower“ - laut der Homepage von Forensic Architecture - den mediterranen Grenzraum hinsichtlich abgesendeter Notrufe über das Alarm Phone. Das Alarm Phone nimmt diese Notrufe auf, leitet sie dann an umliegende Schiffe weiter, die laut Seerecht dazu verpflichtet sind, Search-and-Rescue-Missionen (SAR) zu initiieren. Des Weiteren haben die Agenturen Forensic Architecture und Forensic Oceanography eine fast 30-minütige Rekonstruktion eines Vorfalls eines in Seenot geratenen Gummibootes in der Nacht vom 5 . auf den 6. November 2017 im Seegebiet zwischen Libyen und Lampedusa

18 Siehe dazu die Spots Profiting from misery - how smugglers bring people to Europe und Frontex-coordinated Operation Triton auf dem YouTube-Kanal von FRONTEX. 
auf ihre Homepage gestellt: Auf Basis von Videoaufnahmen von Sea Watch wird hier widerrechtliches Verhalten verschiedener Grenzkontrollvehikel im sogenannten ,Kampf gegen den Menschenhandel ${ }^{\star}$ - ein Schiff der lybischen Küstenwache, ein portugiesisches Flugzeug und ein französisches Schiff über eine 3D-Grafik simuliert. Für Friese $(2014,191)$ entgehen jedoch diese Projekte kaum einer „Topik der Denunziation“ - sind also Teil der Politik der Sichtbarmachung des militärisch-humanitären Komplexes. Denn hier verzahnen sich die repressive Überwachung von migratorischen Bewegungen mit der Gegenüberwachung der Überwachenden zu einem engmaschigen Netz der Versicherheitlichung.

Der ethisch stark aufgeladene Begriff der Denunziation, den Friese hier wählt, muss problematisiert werden, da zivile Seenotrettung trotz zunehmender Kriminalisierung Leben rettet - und somit den humanitären Auftrag ,Europas“ ernst nimmt. ${ }^{19}$ In seiner Überspitzung verweist er allerdings pointiert auf die Gefahr der Kompliz*innenschaft oder Kooptation von zivilgesellschaftlichem Engagement und kritischer Wissenschaft mit bzw. in repressiv-administrative Strukturen, die Migration neoliberal zu managen, ethnonationalistisch einzudämmen und juridico-politisch auf ein Mindestmaß ,erwünschter Migrant*innen` zu beschränken suchen. Insbesondere anti- und postkoloniale sowie queer-feministische Theorien (z.B. Fanon 1985 [1952], 67; Butler 2006, 19) haben auf diese Problematik aufmerksam gemacht und die Bedeutung kritischer Reflexion der eigenen Position betont. Sie verweisen einerseits auf die Verwobenheit in rassifizierte und vergeschlechtlichte Strukturen und andererseits die Notwendigkeit, diese bloßzulegen. In Bezug auf das europäische Grenzregime und dessen asymmetrische Machtverhältnisse, wie sie in rassifizierten Konstellationen präsent sind, muss selbstkritisch gefragt werden, inwiefern die Rettung ,nichtweißer

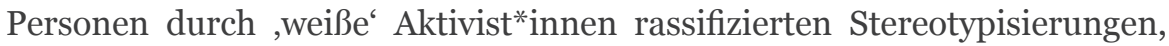
z. B. der Hilflosigkeit und Viktimisierung, unterliegt - und damit einen depolitisierten eurozentrischen Human(itar)ismus reproduziert, der ontologisch-essentialisierende Kategorien des ,bloßen“ Menschseins aufruft. D.h. dass für NGOs und Wissenschaftler*innen des globalen Nordens die Gefahr

19 Um auch auf die widerständige Dimension von WatchtheMed und Alarm Phone hinzuweisen, verweise ich auf einen Aufsatz von Simon Sontowski (2017), in dem die Arbeit von Alarm Phone als Produzent von "Gegen-Narrativen“ und als „politisches Projekt“ beschrieben wird, das sich „Kämpfen um Bewegungsfreiheit verschreibt und gezielt in das Spannungsverhältnis zwischen Grenzschutz und Seenotrettungspflicht interveniert" (Sontowski 2017, 112). 
besteht, einem white saviorism anheimzufallen, in dem sich rassifiziert-vergeschlechtlichte Stereotype als neokoloniale Macht- und Herrschaftsverhältnisse fortschreiben (von Bieberstein und Evren 2016, 472; Spivak 1988, 9off.)..$^{20}$

\section{Zur materiellen Kultur der Grenze}

In den südlichen Regionen Europas ist eine räumliche Ausdehnung der Grenze im Sinne ihrer zunehmenden Überwachung und Versicherheitlichung zu beobachten (Huke et al. 2014). Paradigmatisch für den humanitär-militärischen Komplex steht die spanische Exklave Melilla, die eine Landgrenze zwischen Marokko und Spanien, d.h. der EU, aufweist. ${ }^{21}$ Da man durch die Landgrenze die gefährliche Überfahrt über das Mittelmeer vermeiden kann, rückte dieser spanisch-marokkanische bzw. europäisch-nordafrikanische Grenzraum in die Aufmerksamkeit von Migrant*innen bei der Planung ih-

20 Diese Schleife muss auch von der Autorin gezogen werden, die aus einer akademischen, europäischen und ,weißen' Perspektive diesen Aufsatz verfasst. Diese Verwobenheit kann ich nicht auflösen. Deshalb soll es gerade nicht darum gehen, die Anderen darzustellen und zu vertreten (Spivak 1988, 7off.), sondern zu analysieren, wie ,Europa' diese Anderen konstruiert. Da ich mich nicht in den Anderen hineinversetzen kann - entgegen aller erkenntnistheoretischen Überlegungen im Rahmen von Anerkennungsverhältnissen von Hegel über Habermas bis Honneth -, werde ich mir nicht anmaßen, den Anderen nachvollziehen, gar verstehen zu können. Vielmehr möchte ich als europäisch-deutsche Wissenschaftlerin herausarbeiten, wie Rassifizierung und Vergeschlechtlichung in Europa in einem spezifischen Diskursfragment miteinander verflochten und welche Mechanismen und Prozesse in dieser VerAnderung wirksam werden. Siehe dazu die Organisation No White Saviors (https://nowhitesaviors.org/).

Melilla ist eine spanische Stadt an der nordafrikanischen Mittelmeerküste mit einer Landgrenze zu Marokko und seit 1497 in spanischem Besitz - auch nach der Unabhängigkeit Marokkos von den Kolonialmächten Frankreich und Spanien (1956). Damit gehört Melilla zwar zur Europäischen Union, ist aber von ihrem Zollgebiet ausgenommen und aus dem Schengen-Raum ausgeschlossen. Daher gibt es hier eine immer noch stark bewachte und gut ausgestattete Grenzkontrolle. Melilla ist Teil der westlichen Mittelmeer-Route, über die Migrant*innen über Land in die EU gelangen können und keine gefährliche Überfahrt über das Mittelmeer riskieren müssen - wenngleich es auch eine Seeroute nach Melilla gibt. Sobald es Migrant*innen über die Zaunanlage geschafft haben, befinden sie sich auf EU-Gebiet. Vgl. Soto Bermant 2017 sowie Sidibé, Siebert und Wagner 2016. 
rer Migrationsroute. Die Verräumlichung der Grenze materialisiert sich hier in einer technologisch aufgerüsteten und komplexen Zaunanlage, die nicht nur eine konkrete Linie absichert, sondern einen gesamten Raum, der aus mehreren Zäunen und Stacheldrahtvorrichtungen besteht und mit Überwachungstechnologien - Kameras, Bewegungssensoren, zurückfedernden Anti-Sprung-Vorrichtungen - ausgerüstet ist.

Doch die Verräumlichung der EU-Außengrenze hat eine weitere Funktion: Nicht nur die militärisch-technologische Aufrüstung der Grenze wird legitimiert, sondern auch bestimmte Praktiken der Grenzsicherung, die gegen internationales Recht verstoßen, sind schwieriger zu verfolgen. Hier ist keine Intention zu unterstellen, aber das Ergebnis der Verräumlichung ist eine schwierigere Dokumentation von sogenannten push backs. Im Grenzraum Melillas bleibt sowohl für Grenzbeamt*innen, Migrant*innen als auch Menschrechtsorganisationen unklar, wo genau Spanien beginnt und Marokko endet: wo also EU-Territorium betreten wurde und damit ein Recht auf Beantragung von Asyl besteht und Personen daher nicht zurückgestoßen werden dürfen. In einem Interview mit VICE bestätigt ein spanischer Grenzbeamter, dass er nicht wisse, wo Spanien, d.h. die EU, beginne. Der Beamte suggeriert, dass dieses Unwissen gewollt ist, da dadurch push backs nicht nachgewiesen bzw. rechtlich verfolgt werden könnten (VICE 2015). ${ }^{22}$

Die Überwindung der Zaunanlage Melillas durch den ,Sprung' stellt die günstigste Variante dar, die EU zu erreichen. ${ }^{23}$ Der ,Sprung nach Europa gilt außerdem als weniger gefährlich als eine Überquerung des Mittelmeers. Jedoch können Personen beim ,Sprung‘ lebensbedrohlich verletzt werden. Wie viele Personen jedes Jahr beim ,Sprung' sogar sterben, bleibt undoku-

22 In der EU gilt das sogenannte Non-refoulement-Gebot (GFK, Art. 33, Abs. 1), das bestimmt, dass Personen, sobald sie das Territorium eines Staates betreten haben, nicht mehr zurückgestoßen („push back“, „refoulement“) werden dürfen, sondern ein Anrecht auf ein migrations- bzw. asylrechtliches Verfahren haben. In Melilla gibt es jedoch seit Jahren immer wieder Berichte durch NGOs und Migrant*innen, dass gegen das Verbot des refoulement regelmäßig verstoßen wird. Das Nichtwissen der Beamt*innen über den genauen Grenzverlauf lässt daher vermuten, dass sie bzw. die EU davor ,geschützt' werden soll(en), wegen eines Verstoßes gegen das non-refoulement belangt zu werden.

23 Eindrucksvoll werden die körperlichen, mentalen und auch materiellen Vorbereitungen für diesen ,Sprung' in die EU in der Dokumentation Les Sauteurs - Those Who Jump von Abou Bakar Sidibé, Moritz Siebert und Estephan Wagner (2016) dargestellt. 
mentiert und damit diese Toten auch unsichtbar. Personen, die etwas mehr Geld an die lokalen Grenzüberwindungsnetzwerke zahlen können, nutzen den alltäglichen ,kleinen Grenzverkehr' Melillas: Sie lassen sich in Autokarosserien einarbeiten - im Kotflügel oder unterhalb des Motors -, um die Grenzkontrolle unentdeckt passieren zu können. Angesichts der hohen Temperaturen, die in Melilla erreicht werden können und die Temperatur, die der Motor des Autos hervorbringt, ist auch dieser Weg über die Grenze lebensgefährlich. Manchmal befinden sich Personen stundenlang in den Autokarosserien, wenn z. B. genaue Grenzkontrollen durchgeführt werden. Um die Personen in den Karosserien aufzuspüren - also sichtbar zu machen -, verwenden spanische Grenzbeamt*innen am Grenzkontrollposten der Stadtgrenze Detektoren, die Herzschläge orten können: Nachdem die sichtbaren Insass*innen eines Autos gebeten wurden auszusteigen, wird, ähnlich einem Stethoskop, das Gerät an die Karosserie eines ,verdächtigen' Wagens gehalten. Sind noch Herztöne zu orten, die ein Auto ohne Insass*innen nicht haben kann, wird die Karosserie auseinandergenommen (VICE 2015).

Es ergibt sich also im europäischen Grenzregime eine zweifache paradoxe Situation: Zum einen sind durch die Verräumlichung Grenzen weniger sichtbar, zugleich aber bedeutsamer und strikter zu versicherheitlichen. Während das Grenzregime der EU in seiner Binnenstruktur Grenzen zum Verschwinden bringt (Schengen), avancieren die Außengrenzen zu umkämpften Zonen von Zugehörigkeit, in denen nur für bestimmte Gruppen Mechanismen der Identifikation wirksam werden. Zum anderen werden veranderte Personen durch die technologische Aufrüstung der Grenze hochgradig visibilisiert, z. B. durch Identifikationstechniken, während sie jedoch auch radikal invisibilisiert sind, z. B. in push backs und als nichtbetrauerbare Tote an der Zaunanlage. Die nüchtern-technologischen Maßnahmen der Verräumlichung sind dabei hochgradig affektiv aufgeladen. An den Intersektionen von Hautfarbe, Geschlecht und Alter greift im Grenzraum eine zweifache Demarkation: Auf der einen Seite wird über die rassifizierte phänotypische Erscheinung Nichtzugehörigkeit (diskursiv) festgelegt, so z. B. im Verfahren des racial profiling (Schwarz 2017). Diese identitätspolitische Demarkation wird in migrationspolitische Bestimmungen und Recht übersetzt, die im europäischen Grenzregime immer auch mit sicherheits- und innenpolitischen Erwägungen verschränkt werden. Auf der anderen Seite erfolgt über die Festlegung der Nicht-Zugehörigkeit auch der Ausschluss des fremden Anderen aus dem soziopolitischen Selbst: Über rechtliche Regelungen, wie Staatsbürger*innenschaft, kann Zugehörigkeit nicht erlangt werden, da 
der illegalisierte Übertritt(sversuch) Migrant*innen zu personae non gratae stilisiert, durch die sie ihr Recht auf politische Teilhabe verwirkt haben. Durch die rassifizierten Strukturen der Gesellschaft kann Zugehörigkeit nicht erlangt werden, da Migrant*innen bis zu einem gewissen Grad die fremden Anderen bleiben. Migrant*innen erfahren dann weder in institutionalisierten Rahmungen noch im „Bezugsgewebe menschlicher Angelegenheiten“ (Arendt 2007 [1967], 222) Sichtbarkeit: Entgegen seinem Versprechen von Gleichheit und Freiheit, verweigert ,Europa' (illegalisierten) Migrant*innen - die es ohne die juridico-territoriale Grenzziehung nicht gäbe - eine Teilhabe am soziopolitischen Leben Europas. Im Umkehrschluss kann politische Partizipation durch Migrant*innen nur im Modus des ebenfalls illegalisierten Protests stattfinden. Durch die Illegalisierung des Protests, der nicht innerhalb des Rahmens der repräsentativen Demokratie stattfinden kann, wird dieser auch illegitimisiert. Dadurch kann Protest durch die Mehrheitsgesellschaft nicht als solcher gelesen werden. Er wird daher als ,Chaos' oder ,Aufstand' interpretiert und diskreditiert. In Rekurs auf Butler kann man schlussfolgern, dass die (diskursive) Macht des Rahmens nicht zulässt, dass diese Form des politischen Widerstands als legitimer, demokratischer Protest gelesen wird.

Hier zeigt sich jedoch die Unterscheidung von Dudziak und Volpp: Dass Grenzräume nicht nur eine repressive Funktion erfüllen, sondern auch eine widerständige - in ihren Worten befreiende - Dimension haben, durch die soziokulturelle Imaginationen der Fixiertheit und der Eindeutigkeit herausgefordert werden. Migrant*innen sind nicht nur Opfer repressiver Migrationspolitik, sondern erheben sich selbst zu Akteur*innen des Grenzraums, die repressive Grenzziehungen subvertieren und rassistische Normalisierungen aufdecken und kritisieren (Hess et al. 2017b). Auch dies wird besonders gut an der Zaunanlage Melillas deutlich: In den sogenannten stand offs geht es geflüchteten Personen nicht um den Sprung über die Zaunanlage, sondern darum, möglichst lange auf einem der Zäune auszuharren, um z. B. Journalist*innen die Möglichkeit zu geben, den Protest der Migrant*innen gegen eine repressiv-regressive Migrationspolitik, die sich an der Zaunanlage Melillas materialisiert, zu dokumentieren. Der Protest erhält dadurch mediale Aufmerksamkeit und damit auch Sichtbarkeit. Die „Ungebundenheit“ der illegalisierten Migrant*innen, so zeigt Julia Schulze Wessel $(2017,118)$ in ihrer „politischen Theorie des Flüchtlings“, kann sie in eine „Lage des Sich-Entziehens, des Widerstehens [...] gegen das souveräne Verfügen über [ihre] Kontrolle, Abschiebung oder [...] Abweisung“ versetzen. 
Ohne die multidimensionale Prekarisierung, denen Migrant*innen im und jenseits des Grenzraums ausgesetzt sind, zu ignorieren und ihre „Ungebundenheit“ romantisieren zu wollen, kann der Grenzraum zu einer „Zone[.] der Klandestinität“" (Oberprantacher 2016, 313) und des Widerstands avancieren. So lässt sich die „Grenzüberschreitung“ (Gebhardt 2017, 170) als Akt politischer Subjektivierung verstehen, die einem neoliberalen Versicherheitlichungsmanagement ebenso entgegentritt wie einer ethnonationalistischen Verbarrikadierung oder einer humanitär-moralisierenden Viktimisierung.

Während Mbembe im Konzept der Vergrenzung, im Anschluss an Foucault, die gouvernementale Regierung des Grenzraums durch den militärisch-humanitären Komplex hervorhebt, muss jedoch, wie bei Dudziak und Volpp, aber auch bei Schulze Wessel $(2017,119)$, betont werden, dass er gerade durch seine Uneindeutigkeit ein Raum der Kontestation werden kann. Mit Foucault selbst $(1983,116 f$.) kann man dies auf die fast physikalische Formel bringen, dass dort, wo es Macht gibt, auch Widerstand entsteht. Dieser Widerstand wird im Grenzregime der EU durch Subjektpositionen geleistet, die sich aus ethico-politischen Gründen gegen eine repressive Abschottung der vielzitierten ,Festung Europa' wehren. Er wird jedoch auch von illegalisierten Subjektpositionen geleistet, die weder einer privilegierten, europäischen Linken noch einer humanitär informierten, grünen und urbanen Wohlstandsschicht angehören, sondern unter hegemonialen Bedingungen kaum in Erscheinung treten: (illegalisierte) Migrant*innen. Die Demonstrationen, Petitionen und räumlichen Besetzungen von Lampedusa in Hamburg, Refugee Tent Actions und VOICE Refugee Forum wie auch die körperlich-stummen Praktiken des Widerstands in den Abschiebe- und Unterbringungslagern - Hungerstreik, Verstümmeln der Fingerkuppen, lip sewing und Selbstmord (Cover 2016, 97-101f.), die vor einem destruktiven Eingriff in die eigene körperliche Unversehrtheit nicht zurückschrecken müssen als Widerstände gegen das europäische „Grenzspektakel“ (De Genova 2017, 3) gelesen werden. Diese Widerstände stellen ihrerseits „Gegenspektakel“ (Délano Alonso und Nienass 2016, 437) dar, die vermögen, dem repressiven Rahmen der soziotechnologischen Grenzversicherheitlichung Risse beizufügen. 


\section{In-/Konklusion}

Was verbindet also die medialen Repräsentationen von Aylan Kurdi, dem überfüllten und vom Kentern bedrohten Flüchtlingsboot, der Karikatur aus Charlie Hebdo und die Versicherheitlichungstechnologien des miliärisch-humanitären Komplexes des europäischen Grenzregimes? In ihnen werden Figurationen aufgerufen, durch die die Anderen regiert und in ihrer Nicht-/Betrauerbarkeit (re-)produziert werden. In ihnen wird das Motiv des Ertrinkens zweifach aufgerufen: als tragische Todesursache des migrantisch Anderen oder als Bedrohung für Europa, wenn die Ströme und Wellen von Migrant*innen die europäischen Strände erreichen und diese zu überfluten drohen.

Die Rhetorik und der Affekt der Betroffenheit spielen in der medialen Inszenierung des Ertrinkens (eines migrantischen Kindes) eine zentrale Rolle: Allerdings wird nicht nur Betroffenheit und Mitleid für den Anderen hergestellt. Vielmehr kann sich die humanitär-moralische Zuschauer*innenschaft als ,weiße Rettende ‘ heroisieren - man fühlt sich gut, wenn man sich über Unrecht und Barbarei empört. Jedoch wird in den Darstellungen des Anderen auch Nicht-Betrauerbarkeit hergestellt: In der Repräsentation des ,übervollen Bootes ' wie auch in der Karikatur aus Charlie Hebdo wird ein Bedrohungsszenario skizziert, das Europa heimsucht; seine ,Zivilisation` ertränkt. Diese ambivalente Bedrohung - die Bedrohung ,des Migranten` durch den Tod im Meer und die Bedrohung Europas durch ,den Migranten-

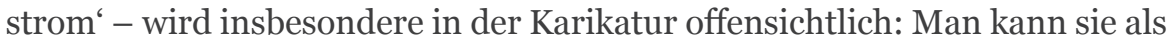
antimuslimischen, rassistischen Kommentar lesen, der den „Sex-Mob-Narrativen“ (Dietze 2016), die auf die Kölner Silvesternacht folgten, bestärkt. Man kann jedoch angesichts anderer Karikaturen, die harsche Kritik am europäischen Grenzregime übten und der generellen politischen Ausrichtung des Satiremagazins, vermuten, dass die Karikatur - wenn auch ethisch fragwürdig - kritisch in den europäischen Migrationsdiskurs nach ,Köln“ intervenieren soll. Gleichwie die Karikatur zu lesen sei, reduziert sie die Betrauerbarkeit Aylan Kurdis, der mit der Gefahr verklebt wird, die von heranwachsenden Männern mit Migrationsbiografie auszugehen scheint. Die visuelle Repräsentation des (jungen, männlich und muslimisch gelesenen) Migranten gleitet zwischen totem Kind und predator - also einem wilden Raubtier und Plünderer. Der Begriff des predator verweist auf eine zügellose, animalische Sexualität: Auch wenn ,Aylan' noch nicht alt genug war, um als ,Mann“ zu gelten, noch nicht alt genug, um als Arschgrapscher eine konkrete Bedrohung für Europa und die ,europäische Frau' darzustellen, 
wird in dieser Karikatur das fremde (kindliche und männlich gelesene) Andere in eine dämonisierend-dehumanisierende Rahmung eingepasst. Diese diskursiv-affektiven Strategien sind dabei nicht die Ausnahme, sondern der Normalfall des europäischen Grenzregimes. Denn die gefährliche ,Raubtiernatur' des Anderen legitimiert schlussendlich seine Tötung. Durch die diskursiv-visuelle Umdeutung des Anderen im metonymischen Gleiten sieht die europäische Zuschauer*innenschaft in der Karikatur nicht mehr das ertrunkene Kind, sondern den hypersexualisierten Anderen, den es einzudämmen und zu beseitigen gilt. Damit gleitet der rezente Migrationsdiskurs Europas metonymisch zwischen Tod und Bedrohung: das Foto des toten Jungen aus Syrien zeigt ein ,Opfer ${ }^{6}$ des europäischen Grenzregimes und doch wird das Bild immer wieder von der Karikatur des arschgrapschenden Migranten heimgesucht, das suggeriert, was Europa droht, wenn ,Aylan Kurdi‘ erwachsen wird. Denn, wie die Süddeutsche Zeitung ihre Leser*innen im Januar 2016 wissen lässt, ,[V]iele junge Muslime können nicht entspannt dem anderen Geschlecht begegnen. Das sind jedesmal hochsexualisierte Situationen. Auch das ist der Boden für den Exzess von Köln" (zit. nach Hark und Villa 2018, 61). Die ethnosexistische Regierung des Anderen in den mediterranen Todeslandschaften des europäischen Grenzregimes, insbesondere in Gestalt des ,schwarzen Mannes', reduziert Betrauerbarkeit maßgeblich. Sie führt schließlich dazu, dass sein Tod nicht betrauert wird, weil er nicht zählt.

\section{Literatur}

Agamben, Giorgio. 2002. Homo sacer. Die Souveränität der Macht und das nackte Leben. Frankfurt a.M.: Suhrkamp.

Ahmed, Sara. 2004. „Affective Economies“. Social Text 22 (2, 79): 117-139. https:// doi.org/10.1215/01642472-22-2_79-117.

Alarm Phone. O.A. Zugegriffen am 27.7.2019. https://alarmphone.org/de/.

Arendt, Hannah. 2007 [1967]. Vita activa. Oder vom tätigen Leben. München, Zürich: Piper.

Athanasiou, Athena, und Simon Sheikh. 2019. „Formations of Political-Aesthetic Criticality. Decolonizing the Global in Times of Humanitarian Viewership“. In Curating after the global. Roadmaps for the present, herausgegeben von Paul O'Neill, Simon Sheikh, Lucy Steeds und Mick Wilson, 76-110. Feldmeilen: Luma Foundation/Annandale-on-Hudson, NY: Center for Curatorial Studies, Bard College. 
Bieberstein, Alice von, und Erdem Evren. 2016. „From Aggressive Humanism to Improper Mourning. Burying the Victims of Europe's Border Regime in Berlin“. Social Research 83(2): 453-479.

Butler, Judith. 2015. Notes toward a Performative Theory of Assembly. Cambridge, MA/London: Harvard University Press.

Butler, Judith. 2016 [2009]. Frames of War. When Is Life Grievable?. London, New York: Verso.

Butler, Judith. 2006. Precarious Life. The Powers of Mourning and Violence. London, New York: Verso.

Castro Varela, María do Mar, und Paul Mecheril. 2016. „Die Dämonisierung der Anderen. Einleitende Bemerkungen“. In Die Dämonisierung der Anderen. Rassismuskritik der Gegenwart, herausgegeben von María do Mar Castro Varela und Paul Mecheril, 7-19. Bielefeld: transcript.

Cigdem, Cenk. 2015. „Ich wollte den verstummten Schrei des Jungen hörbar machen“. Rheinische Post, 3. September 2015. https://rp-online.de/politik/ausland/ toter-junge-von-bodrum-jetzt-spricht-fotografin-niluefer-demir_aid-17588775.

Cover, Rob. 2016. „Suicides of the Marginalised. Cultural Approaches to Suicide, Minorities and Relationality“. Cultural Studies Review 22(2): 90-113. https://doi. org/10.5130/csr.v22i2.4708.

Därmann, Iris. 2020. Undienlichkeit. Gewaltgeschichte und politische Philosophie. Berlin: Matthes \& Seitz.

Délano Alonso, Alexandra, und Benjamin Nienass. 2016. „Deaths, Visibility, and Responsibility. The Politics of Mourning at the US-Mexico Border". Social Research 83(2): 421-451.

Dietze, Gabriele. 2016. „Ethnosexismus. Sex-Mob-Narrative um die Kölner Silvesternacht“. movements 2(1): 178-185.

Dudziak, Mary L., und Leti Volpp. 2006. „Introduction“. In Legal Borderlands. Law and the Construction of American Borders, herausgegeben von Mary L. Dudziak und Leti Volpp, 1-18. Baltimore, MD: The John Hopkins University Press.

Danewid, Ida. 2017. „White innocence in the Black Mediterranean. Hospitality and the erasure of history“. Third World Quarterly 38(7): 1674-1689.

De Genova, Nicholas. 2017. „Introduction. The Borders of ,Europe' and the European Question“. In The Borders of ,Europe‘. Autonomy of Migration, Tactics of Bordering, herausgegeben von Nicholas de Genova, 1-35. Durham, NC / London: Duke University Press.

Europäisches Parlament. 2020. „Raum der Freiheit, der Sicherheit und des Rechts: Allgemeine Aspekte“. Zugegriffen am 21.9.2020. https://www.europarl.europa. $\mathrm{eu} / \mathrm{ftu} / \mathrm{pdf} / \mathrm{de} / \mathrm{FTU}$-4.2.1.pdf.

Fanon, Frantz. 1985 [1952]. Schwarze Haut, weiße Masken. Frankfurt a.M.: Suhrkamp. 
Forensic Architecture. O.A. Zugegriffen am 27.7.2019. http://archive.forensic-architecture.org/news/watchthemed/.

Forensic Architecture. O.A. Zugegriffen am 25.2.2019. https://www.forensic-architecture.org/case/sea-watch/.

Foucault, Michel. 1978. Dispositive der Macht. Über Sexualität, Wissen und Wahrheit. Berlin: Merve.

Foucault, Michel. 1983. Der Wille zum Wissen. Sexualität und Wahrheit I. Frankfurt a.M.: Suhrkamp.

Foucault, Michel. 2015 [1978f.]. Geschichte der Gouvernementalität I + II. Frankfurt a.M.: Suhrkamp.

Frank, Arno. 2015. „Mittelmeer. Mit Wucht durch den Wahrnehmungspanzer“. Die Zeit, 3. September 2015. https://www.zeit.de/kultur/2015-09/fluechtling-mittelmeer-bodrum-toter-junge-syrien.

Friese, Heidrun. 2014. Die Grenzen der Gastfreundschaft. Die Bootsflüchtlinge von Lampedusa und die europäische Frage. Bielefeld: transcript.

FRONTEX. 2016. „Profiting from misery - how smugglers bring people to Europe“. Hochgeladen am 18.2.2016. https://www.youtube.com/watch?v=W7OsRz4Ubeg.

FRONTEX. 2015. „Frontex-coordinated Operation Triton“. Hochgeladen am 23.6.2015. https://www.youtube.com/watch?v=YOF_rh7ZIhI.

Gebhardt, Mareike. 2014. Politisches Handeln in der postmodernen Konstellation. Kritische Demokratietheorie nach Hannah Arendt und Jürgen Habermas. Baden-Baden: Nomos.

Gebhardt, Mareike. 2017. „Grenzverschiebungen. Überschreitung als demokratische Praxis“. In Die Grenzen der Demokratie. Gegenwartsdiagnosen zwischen Recht und Politik, herausgegeben von Annette Förster und Matthias Lemke, 153-180. Wiesbaden: Springer VS.

Gebhardt, Mareike. 2020. „To Make Live and Let Die. On Sovereignty and Vulnerability in the EU Migration Regime“. Redescriptions 23(2): 120-37. http://doi. org $/ 10.33134 /$ rds.323.

Grimaldi, Giuseppe. 2019. „The Black Mediterranean. Liminality and the Reconfiguration of Afroeuropeanness". Open Cultural Studies 3: 414-427.

Hark, Sabine, und Paula-Irene Villa. 2018. Unterscheiden und herrschen. Ein Essay zu den ambivalenten Verflechtungen von Rassismus, Sexismus und Feminismus in der Gegenwart. Bielefeld: transcript.

Heimeshoff, Lisa-Marie, Sabine Hess, Stefanie Kron, Helen Schwenken und Miriam Trzeciak, Hrsg. 2014. Grenzregime II. Migration, Kontrolle, Wissen. Transnationale Perspektiven. Berlin, Hamburg: Assoziation A.

Hess, Sabine und Bern Kasparek, Hrsg. 2010. Grenzregime. Diskurse, Praktiken, Institutionen in Europa. Berlin, Hamburg: Assoziation A. 
Hess, Sabine, Bernd Kasparek, Maria Schwertl und Simon Sontowski. 2015 „Europäisches Grenzregime“. movements 1(1).

Hess, Sabine, Bernd Kasparek, Stefanie Kron, Mathias Rodatz, Maria Schwertl und Simon Sontowski, Hrsg. 2017a. Der lange Sommer der Migration. Grenzregime III. Berlin, Hamburg: Assoziation A.

Hess, Sabine, Bernd Kasparek, Stefanie Kron, Mathias Rodatz, Maria Schwertl und Simon Sontowski, Hrsg. 2017b. „Der lange Sommer der Migration. Krise, Rekonstruktion und ungewisse Zukunft des europäischen Grenzregimes“. In Der lange Sommer der Migration. Grenzregime III, herausgegeben von Sabine Hess, Bern Kasparek, Stefanie Kron, Mathias Rodatz, Maria Schwertl und Simon Sontowski, 6-24. Berlin, Hamburg: Assoziation A.

Huke, Nikolai, Dana Lüddemann und Jens Wissel. 2014. „FRONTEX. Verlängerter Arm der Mitgliedsstaaten und Europäisierungsmotor der Grenzkontrolle“. In Kämpfe um Migrationspolitik. Theorie, Methode und Analysen kritischer Europaforschung, herausgegeben von der Forschungsgruppe Staatsprojekt Europa, 169-186. Bielefeld: transcript.

International Center for Migration Policy Development (ICMPD). O.A. Zugegriffen am 25.2.2019. https://www.icmpd.org/home/.

Jensen, Uffa. 2017. Zornpolitik. Berlin: Suhrkamp.

Kerstin, Daniel, und Marcus Leuoth, Hrsg. 2020. Der Begriff des Flüchtlings. Rechtliche, moralische und politische Kontroversen. Berlin: J.B. Metzler/Springer

Maxwill, Peter. 2015. „Solche Bilder brennen sich in die Netzhaut ein“. Spiegel Online, 3. September 2015. https://www.spiegel.de/kultur/gesellschaft/medienethiker-alexander-filipovic-foto-ist-kaum-auszuhalten-a-1051262.html.

Mbembe, Achille. 2003. „Necropolitics“. Public Culture 15(1): 11-40. Project MUSE.

Mbembe, Achille. 2018. „Sicherheit und Migration. Die große Vergrenzung“. die tageszeitung, 14. Juli 2018. https://taz.de/Sicherheit-und-Migration/!5517629/.

Mecheril, Paul, und Monica van der Haagen-Wulff. 2016. „Bedroht, angstvoll, wütend. Affektlogik der Migrationsgesellschaft“. In Die Dämonisierung der Anderen. Rassismuskritik der Gegenwart, herausgegeben von María do Mar Castro Varela und Paul Mecheril, 119-141. Bielefeld: transcript.

Mohanty, Chandra Talpade. 1984. „Under Western Eyes. Feminist Scholarship and Colonial Discourses“. boundary 2, 12(3): 333-358.

Neuhauser, Johanna, Sabine Hess und Helen Schwenken. 2017. „Unter- oder überbelichtet. Die Kategorie Geschlecht in medialen und wissenschaftlichen Diskursen zu Flucht“. In Der lange Sommer der Migration. Grenzregime III, herausgegeben von Sabine Hess, Bern Kasparek, Stefanie Kron, Mathias Rodatz, Maria Schwertl und Simon Sontowski, 176-195. Berlin, Hamburg: Assoziation A.

No White Saviors. O.A. Zugegriffen am 30.3.2020. https://nowhitesaviors.org/. 
Oberprantacher, Andreas. 2016. „Radikal demokratischer Ungehorsam. ,Illegale“ als strittiges politisches Subjekt“. Zeitschrift für Praktische Philosophie 3(1): 305338.

Oberprantacher, Andreas. 2018. „Wi(e)derständiges Gedenken. Trauer und Klage nach Butler“. In Judith Butlers Philosophie des Politischen. Kritische Lektüren, herausgegeben von Gerald Posselt, Tatjana Schönwälder-Kuntze und Sergej Seitz, 209-231. Bielefeld: transcript.

Parkinson, Joe und David George-Cosh. 2015. „Image of Drowned Syrian Boy Echoes Around World“. The Wall Street Journal, 3. September 2015. https://www.wsj. com/articles/image-of-syrian-boy-washed-up-on-beach-hits-hard-1441282847.

Proglio, Gabriele, Camilla Hawthorne, Ida Danewid, P. Khalil Saucier, Giuseppe Grimaldi, Angelica Pesarini und Timothy Raeymakers, Hrsg. 2020. The Black Mediterranean. Bodies, Borders and Citizenship. London: Palgrave Macmillan.

Reuter, Julia. 2002. Die Ordnungen des Anderen. Zum Problem des Eigenen in der Soziologie des Fremden. Bielefeld: transcript.

Said, Edward W. 1979. Orientalism. New York/NY: Vintage Books/Random House.

Schmitt, Carl. 1974 [1950]. Der Nomos der Erde im Völkerrecht des Jus Publicum Europaeum. Berlin: Duncker \& Humblot.

Schulze Wessel, Julia. 2017. Politische Theorie des Flüchtlings. Bielefeld: transcript.

Schwarz, Nina Violetta. 2017. „Kämpfe um Bewegung in Marokko. Grenzmanagement und Widerstand“. In Der lange Sommer der Migration. Grenzregime III, hrsg. Sabine Hess, Bernd Kasparek, Stefanie Kron, Mathias Rodatz, Maria Schwertl und Simon Sontowski, 61-71. Berlin, Hamburg: Assoziation A.

Schwarzer, Alice. 2017. „Silvester: Geht Ideologie vor Realität?“. EMMA, 4. Januar 2017. https://www.emma.de/artikel/silvester-geht-ideologie-vor-realitaet-333975.

Schwarzer, Alice. 2016. „Was geschah wirklich an Silvester?“. EMMA, 25. Februar 2016. https://www.emma.de/artikel/editorial-von-alice-schwarzer-331581.

Schwennicke, Christoph. 2018. „Altersprüfung bei Flüchtlingen. Gesucht wird kein Geburtstag“. Cicero, 4. Januar 2018. https://www.cicero.de/innenpolitik/alterspruefung-fluechtlinge-minderjaehrige-frank-montgomery-aerztekammer.

Sidibé, Abou Bakar, Moritz Siebert und Estephan Wagner. 2016. „Les Sauteurs - Those Who Jump“. Zugegriffen am 11.3.2020. https://www.youtube.com/ watch?v=KxBo9Rb4-a8.

Smythe, S. A. 2018. „The Black Mediterranean and the Politics of the Imagination“. Middle East Report 286: o.A.

Sontowski, Simon. 2017. „Viapolitische Kämpfe um Bewegungsfreiheit. Das WatchtheMed Alarm Phone und die Krise des europäischen Grenzregimes in der Ägais“. In Der lange Sommer der Migration. Grenzregime III, hrsg. Sabine Hess, Bernd Kasparek, Stefanie Kron, Mathias Rodatz, Maria Schwertl und Simon Sontowski, 97-114. Berlin, Hamburg: Assoziation A. 
Soto Bermant, Laia. 2017. „The Mediterranean Question. Europe and its Predicament in the Southern Peripheries“. In The Borders of ,Europe'. Autonomy of Migration, Tactics of Bordering, herausgegeben von Nicholas de Genova, 120-140. Durham, NC, London: Duke University Press.

Spivak, Gayatri Chakravorty. 1988. „Can the Subaltern Speak?“. In Marxism and the Interpretation of Culture, herausgegeben von Cary Nelson und Lawrence Grossberg, 66-111. Basingstoke: Macmillan Education.

UNHCR. 2019. „Jeden Tag 6 Tote im Mittelmeer“. Zugegriffen am 6.7.2019. https:// www.uno-fluechtlingshilfe.de/informieren/aktuelles/news/uebersicht/detail/ artikel/unhcr-bericht-jeden-tag-6-tote-im-mittelmeer/.

VICE News. 2015. „Storming Spain’s Razor-Wire Fence: Europe Or Die“. Zugegriffen am 11.3.2020. https://www.youtube.com/watch?v=OmqOlxNQABI.

WatchtheMed. O.A. Zugegriffen am 27.7.2019. https://watchthemed.net/.

Zentrum für Politische Schönheit. o.A. „Die Toten kommen.“ Zugegriffen am 15.9.2020. https://politicalbeauty.de/toten.html.

Zentrum für Politische Schönheit. O.A. „Die Schande von Melilla. Schematische Darstellung der spanischen EU-Außengrenze“. Zugegriffen am 23.9.2020. https:// politicalbeauty.de/mauerfall.html. 